Check for updates

Cite this: RSC Adv., 2017, 7, 18384

\title{
Synthesis of new conjugated polymers with coordinated praseodymium complexes for polymer memory devices $\dagger$
}

\author{
Wei Lv, Huijiao Liu, Wen Wang, * E. Yang, Hongyu Zhen and Qidan Ling (DD* \\ A series of novel praseodymium(Pr)-coordinated polymers with fluorene, phthalimide and bipyridine \\ moieties in the main chain were synthesized via a coordination reaction and palladium-catalyzed Suzuki \\ coupling polycondensation. Their structures, optical features and memory performance have been well \\ studied. A resistive switching device with the configuration of ITO/polymer/Al was constructed using \\ a spin-coating process. The device exhibits nonvolatile write-once-read-many-times (WORM) memory \\ behavior. Based on the electrochemical properties and theoretical calculations of the polymers, the \\ effects of the phthalimide moiety and neutral Pr complex on the polymer memory device performance \\ were investigated. Our work offers valuable clues on the development of polymer memory devices.
}

Received 27th December 2016

Accepted 17th March 2017

DOI: $10.1039 / \mathrm{c} 6 \mathrm{ra28757c}$

rsc.li/rsc-advances

materials and devices, which can avoid the problem of phase

\section{Introduction}

Along with dramatically increased capacity and the drastically decreased size of semiconducting memory devices in recent decades, there is a growing demand for exploiting newgeneration data-storage technology. Compared with traditional inorganic materials, organic materials are promising candidates for the next generation of memory applications, which also provide many advantages such as low power consumption, good scalability, large capacity and structural tenability. ${ }^{1}$

Different from capacitor and transistor memory devices, a resistor-type memory device does not require a specific cell structure or to be integrated with the CMOS technology. ${ }^{2}$ The resistor-type memory devices offer simplicity in fabrication and allow possibility for high data storage density via two dimensional, or even three-dimensional (stacking) cross-bar arrays. ${ }^{3}$ Several kinds of memory materials have been developed such as organic small-molecular compounds, polymers, inorganic/ organic hybrid materials, polymer hybrid materials and organometallic materials. ${ }^{4,5}$ Compared with small organic molecules, polymers have excellent film-forming ability and can easily form thin film on the substrates from a solution. In the pioneering studies of polymer resistive memory devices, the doped or blended polymer systems, namely polymer composites, are often used as the active materials. ${ }^{6-8}$ Most recently, much attention have been paid to single polymer-based memory

College of Chemistry and Chemical Engineering, College of Materials Science and Engineering, Fujian Key Laboratory of Polymer Materials, Fujian Normal University, Fuzhou 350007, China.E-mail: wangwen@fjnu.edu.cn; qdling@fjnu.edu.cn

$\dagger$ Electronic supplementary information (ESI) available. See DOI: $10.1039 / \mathrm{c} 6 \mathrm{ra} 28757 \mathrm{c}$ separation and exhibit promising application for nextgeneration memory devices. ${ }^{9,10}$

Metal complexes can exhibit special optoelectronic properties due to the rich electronic structures of metal centers and ligands, such as the highly efficient phosphorescence, good redox reversibility and electro-accepting ability, which have been widely applied in organic light emitting diodes. ${ }^{5}$ Organometallic materials including organometallic small-molecule and polymer containing metal complexes also exhibit the promising application in memory devices. Up to now, a variety of metal complex-containing organic materials have been synthesized and used for memory devices, such as polymers containing Eu, ${ }^{11,12} \mathrm{Cu},{ }^{13} \mathrm{Ir},{ }^{14-16} \mathrm{Pt},{ }^{17,18} \mathrm{Co},{ }^{19} \mathrm{Fe}$ (ferrocene), ${ }^{20,21}$ $\mathrm{Ru}^{22}$ complexes and organometallic materials containing $\mathrm{Rh},{ }^{23}$ $\mathrm{Au}^{24,25}$ graphane oxide containing $\mathrm{Fe}\left(\right.$ ferrocene) complexes. ${ }^{26}$ Although good advances have been made in this field, compared with other organic memory materials, polymers containing metal complexes memory materials and devices are still at an early stage, so it is important to exploit and investigate new polymer materials containing metal complexes.

In general, there are two kinds of methods to synthesize organometallic polymer. One is synthesis of polymer chains containing the ligand group first and subsequent incorporation of metal complexes into the polymers, which might exists the uncertainty of metal complexes content and the steric hindrance problem of coordination reaction. Another is synthesis of metal-containing monomers followed by polymerization of reactable monomers, which might encounter the problem of synthesis of metal-containing monomers and lower reactivity. It is well known that lanthanide metal triisopropyloxide with small polarity could easily coordinate the ligand with the $N$-heterocyclic aromatic structure. ${ }^{27-29}$ Lanthanide metal 
triisopropyloxide-containing polymers can be obtained by fewer synthetic steps. As demonstrated in reported work, lanthanide metal triisopropyloxide could obviously affect the optoelectronic properties of the polymer. ${ }^{29,30}$ Compared with above organometallic polymer, the role of introducing lanthanide metal triisopropyloxide on the memory devices has been rarely reported.

Electron donor-acceptor (D-A) polymers are considered as suitable resistor-type memory materials because of charge transfer (CT) between the donor and accepter moieties. ${ }^{31}$ The memory characteristics based on the $\mathrm{D}-\mathrm{A}$ polymer can be adjusted over a wide range through the modifications on polymer structures. ${ }^{9}$ By introducing proper electron-acceptor moiety into the conjugated polymers such as polyfluorene, or polycarbazole, the optoelectronic properties and molecular-stacking of the polymer might be affected thereby obtaining different memory effects. ${ }^{5}$ Based on the above, we designed and synthesized a series of new conjugated polymers containing fluorene moiety, phthalimide $(\mathrm{Ph})$ moiety with proper electron-accepting ability, bipyridine (Bipy) moiety and neutral Pr complexes with low cost by Suzuki coupling reaction and coordination reaction.

First, Bipy unit was introduced into the conjugated polymer backbone containing fluorene moiety and phthalimide moiety as coordination site. Second, Pr-coordinated conjugated polymers were prepared by coordination of the neutral Pr triisopropyloxide and polymers, in which neutral Pr triisopropyloxide was surrounded by enormous polymer chain. Here, Pr was selected due to lower cost and the smaller molecular weight. The Pr content in the polymer can be easily adjusted by changing the proportion of the Bipy unit. To the best of our knowledge, neutral Pr-coordinated polymers have not been used in the organic memory devices.

In order to explore the effect of the Pr triisopropyloxide on optoelectronic properties, the UV-vis absorption, photoluminescence (PL), electrochemical properties and memory device performance of the resulting polymers were studied. By controlling the content of Pr, the memory performance could be tuned and improved. Meanwhile, the effect of Ph moiety on the device performance was explored.

\section{Experimental}

\section{Materials}

All the reagents and solvents used in this study were purchased from Sinopharm Chemical Reagent Co. and used without further purification.

\section{Measurements}

${ }^{1} \mathrm{H}$ NMR spectra data were expressed in parts per million relative to the internal standard and were obtained on a BRUKER AVIII-400 NMR spectrometer. Pr content was determined by ash method and EDTA (ethylenediaminetetraacetic acid disodium salt) titration. UV-vis absorption spectra in thin film were obtained on a SHIMADZU UV-2600 spectrometer. The photoluminescence spectra were recorded on a SHIMADZU RF5301PC. Molecular weight and polydispersity of the polymer were determined by gel permeation chromatography (GPC) analysis with polystyrene standard calibration (Waters high pressure GPC assembly model 1515 pump, refractive index detectors, solvent THF). Mass spectra were obtained on a Bruker microflex MALDI-TOF/TOF mass spectrometer. Cyclic voltammetry (CV) measurements were conducted on a CHI600D electrochemical analyzer using a three-electrode cell under $\mathrm{N}_{2}$ atmosphere. The polymer films on a glassy carbon electrode (working electrode) were scanned anodically and cathodically in a solution of tetrabutylammonium hexafluorophosphate ( $n$ $\left.\mathrm{Bu}_{4} \mathrm{NPF}_{6}\right)$ in acetonitrile $(0.1 \mathrm{M})$ with $\mathrm{Ag} / \mathrm{AgCl}$ and a platinum wire as the reference and counter electrode, respectively. The experiments were calibrated with the standard ferrocene/ ferrocenium (FOC) redox system and assumption that the energy level of FOC is $4.8 \mathrm{eV}$ below vacuum, and the value of $0.38 \mathrm{~V}$ was found for FOC $v s$. Ag/AgCl. The energy levels of the highest occupied molecular orbital (HOMO) and the levels of lowest unoccupied molecular orbital (LUMO) of the polymers were calculated using the following equations: $E_{\text {номо }}=$ $-\left(E_{\mathrm{ox}}^{\mathrm{onset}}+4.42\right) \mathrm{eV}, E_{\mathrm{LUMO}}=E_{\mathrm{HOMO}}+E_{\mathrm{g}}^{\mathrm{opt}}$, respectively, where $E_{\mathrm{ox}}^{\text {onset }}$ was the onset potential for oxidation relative to the $\mathrm{AgCl} /$ $\mathrm{Ag}$ reference electrode, $E_{\mathrm{g}}^{\mathrm{opt}}$ was the optical band gap of the polymer.

\section{Fabrication and characterization of memory devices}

The memory devices were fabricated with the configuration of ITO/polymer/Al. The ITO-coated glass substrate was precleaned by ultrasonication with water, acetone, and isopropanol each for $20 \mathrm{~min}$. The polymers were dissolved in chlorobenzene at a concentration of $10 \mathrm{mg} \mathrm{mL}^{-1}$ and spin-coated onto the ITO substrate at a speed of $1300 \mathrm{rpm}$ for $60 \mathrm{~s}$. The films thickness were about $45-50 \mathrm{~nm}$ measured by the Stylus Profilometer. Finally, Al top electrode with a thickness of $150 \mathrm{~nm}$ was deposited onto the polymer film surface through a shadow mask by thermal evaporation under vacuum. The electrical characterization of the memory devices was performed by a KEYSIGHT B1500A semiconductor device analyzer equipped with a KEYSIGHT 16440A SMU/Pulse generator.

\section{Theoretical calculations}

All calculations were carried out with the Gaussian 09 package. ${ }^{32}$ The optimization of the complex structure was performed using B3LYP density functional theory. Because orbitals do not play a major role in Pr-ligand bonds, ${ }^{33}$ we used the large-core quasirelativistic effective core potential (ECP) with $46+4 \mathrm{f}^{\mathrm{n}}$ electrons for Pr, as reported by Dolg et al. ${ }^{34}$ For valence orbitals, the $(7 \mathrm{~s} 6 \mathrm{p} 5 \mathrm{~d}) /[5 \mathrm{~s} 4 \mathrm{p} 3 \mathrm{~d}]$ basis set was used. For all other atoms, the 6$31 \mathrm{G}$ basis set was used. The contours of the HOMO and LUMO orbitals were plotted.

\section{Synthesis of the monomers and polymers}

The monomer 9,9'-dioctylfluorene-2,7-bis(boronic acid pinacol ester) was synthesized according to literature method. ${ }^{29}$ Phthalimide monomer was prepared in two steps according to literature method. ${ }^{35}$ 
A general procedure of polymerization was used for the synthesis of the Pr-free polymers (PFPh, PFPh(Bipy) ${ }_{n}$ ). Under the protection of nitrogen, 9,9'-dioctylfluorene-2,7-bis(boronic acid pinacol ester), $N$-(2-ethyl hexyl)-3,6-dibromom-phthalimide,

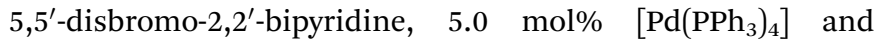
toluene, 2 mol L${ }^{-1}$ aqueous solution of $\mathrm{K}_{2} \mathrm{CO}_{3}$ and Aliquat 336 as phase transfer catalyst were added to a round-bottom flask. The mixture was vigorously stirred at $110{ }^{\circ} \mathrm{C}$ for $72 \mathrm{~h}$. Bromobenzene was dropped to react for $6 \mathrm{~h}$ and phenylboronic acid was dropped to end-cap the polymer for $6 \mathrm{~h}$. After the mixture was cooled to room temperature, it was slowly added dropwise to methanol. A fibrous solid was obtained by filtration. The solid was washed in a Soxhlet extractor with methanol and acetone for $3 \mathrm{~d}$ to remove oligomers and catalyst residues. The resulting polymers were collected and dried under vacuum.

The following procedure was adopted for the synthesis of $\mathrm{Pr}$ coordinated polymers (PFPh(BipyPr $\left.)_{n}\right)$.

Pr triisopropoxide was synthesized from anhydrous $\mathrm{Pr}$ chloride (Scheme 1). The isopropoxy groups of the salt can be easily substituted by chelators to form a Pr complex in anhydrous organic solvents.

Under a nitrogen atmosphere, polymer (50 mg), Pr triisopropoxide and toluene were mixed together in a round-bottom flask. After the solid was dissolved, the solution was refluxed for $1 \mathrm{~d}$. The reactant was cooled down to room temperature and was poured into methanol and filtered. The crude product was extracted by Soxhlet extractor with chloroform. The fraction from chloroform was concentrated under reduced pressure and precipitated into methanol. The resulting polymer was collected and dried under vacuum.
The synthetic details and characterization data, such as ${ }^{1} \mathrm{H}$ NMR, UV-vis absorption spectra and PL spectra diagrams of the polymers are included in the ESI. $\dagger$

For comparison, the polymer $\mathrm{PF}(\mathrm{BipyPr})_{6}$ without $\mathrm{Ph}$ moiety was also synthesized by similar reactions. The synthetic route, details and characterization data are included in the ESI. $\dagger$

\section{Results and discussion}

\section{Synthesis and characterization}

The synthetic routes are shown in Scheme 1. A general procedure of Suzuki coupling polymerization was used for the synthesis of the Pr-free polymers (PFPh, PFPh(Bipy) $)_{n}$ ). Pr triisopropoxide was synthesized from anhydrous $\mathrm{Pr}$ chloride (Scheme 1). The Pr-coordinated polymers $\left(\mathrm{PFPh}(\mathrm{BipyPr})_{n}\right)$ were prepared by coordination reaction with the Pr-free polymer and Pr triisopropoxide. The feed ratios of Pr complex in the polymers were 4, 6, 8 and $10 \mathrm{~mol} \%$, and the corresponding polymers were named $\mathrm{PFPh}(\text { BipyPr })_{4}, \quad \mathrm{PFPh}(\text { BipyPr })_{6}, \quad \mathrm{PFPh}(\text { BipyPr })_{8}$, $\operatorname{PFPh}(\text { BipyPr })_{10}$, respectively.

Fig. 1 shows the ${ }^{1} \mathrm{H}$ NMR spectra of $\mathrm{PFPh}, \operatorname{PFPh}(\mathrm{Bipy})_{n}$ and $\operatorname{PFPh}(\text { BipyPr })_{n}$. The signals of 8.0-10.0 ppm correspond to the protons on the Bipy moieties, while the peaks at $\delta=7.0-$ $7.9 \mathrm{ppm}$ are due to the aromatic protons of fluorene moieties and phthalimide moieties. The peaks at $\delta=3.55$ and 2.07$0.80 \mathrm{ppm}$ are attributed to the alkyl chain of phthalimide moieties and fluorene moieties. As the content of the Bipy units increases, the proton NMR spectra exhibit a corresponding enhancement in the intensity of the peaks associated with Bipy, such as the peaks at about $\delta=9.07,8.58,8.16 \mathrm{ppm}$ belonging to

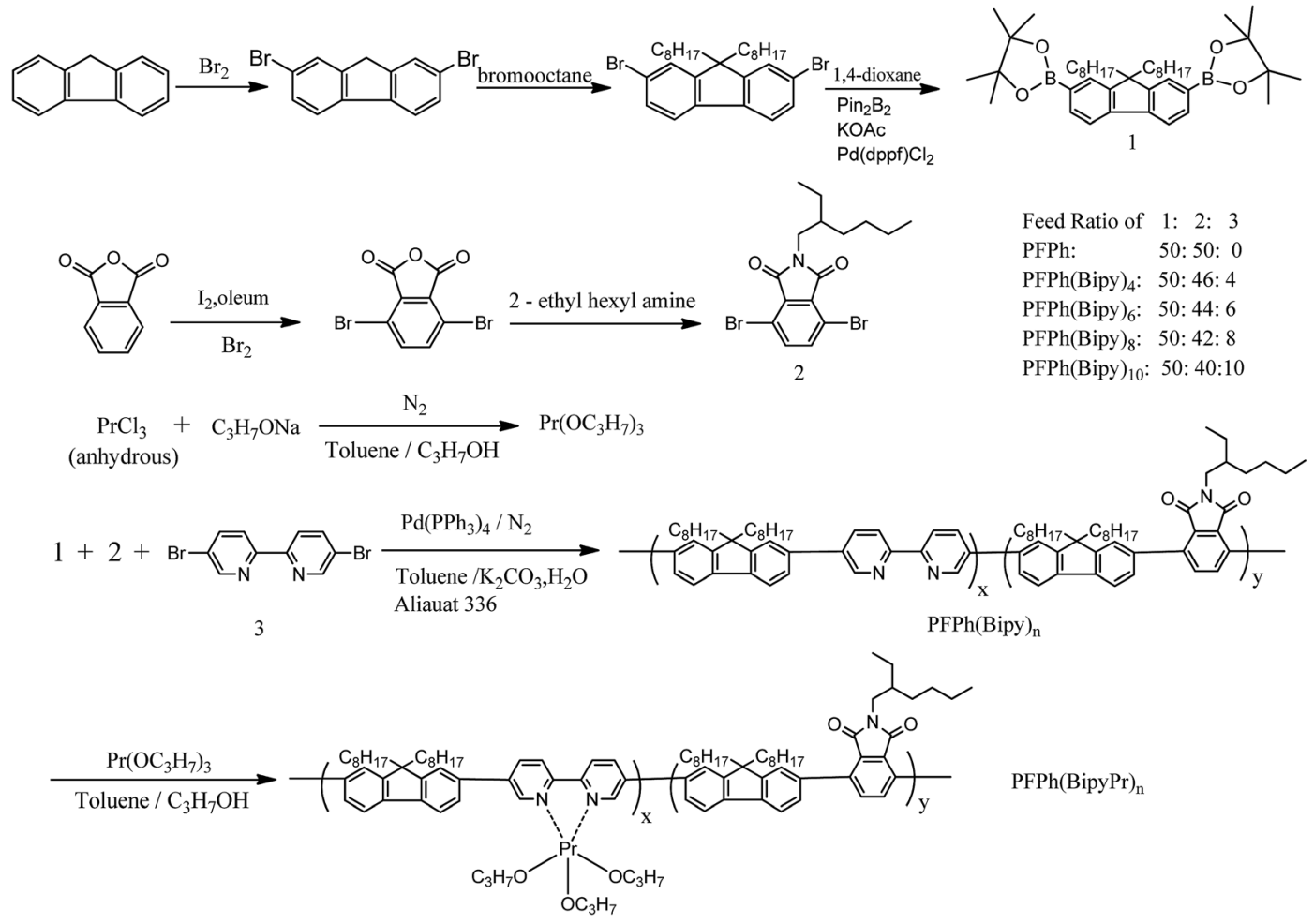

Scheme 1 Synthetic routes and molecular structures of the polymers. 

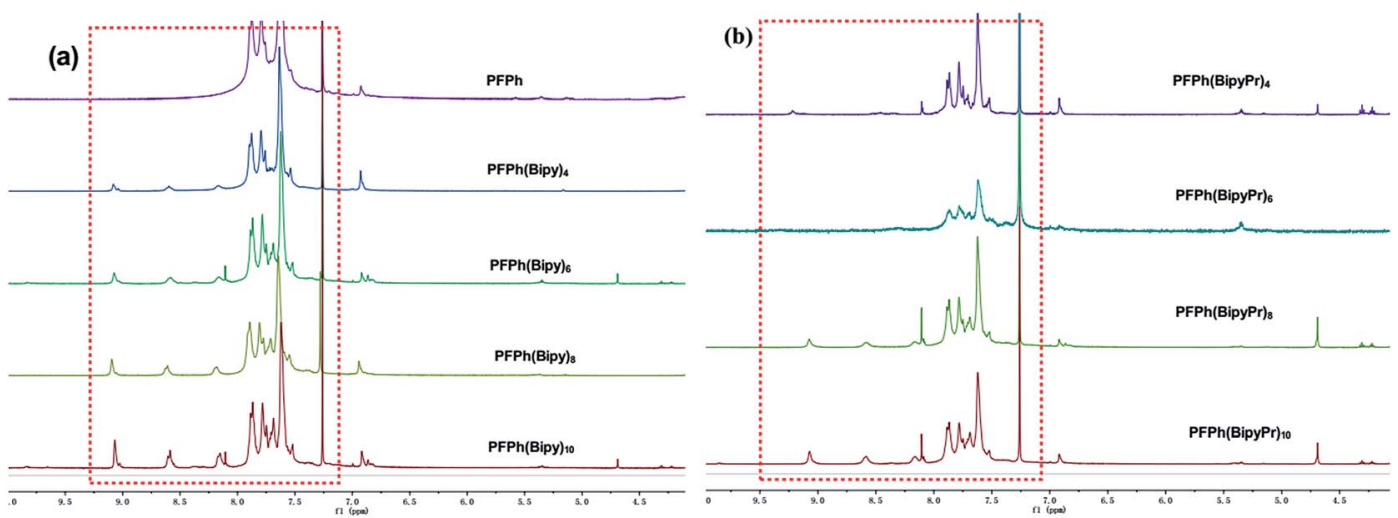

Fig. $1{ }^{1} \mathrm{H}$ NMR spectra of the polymers: (a) PFPh, PFPh(Bipy) ${ }_{n}$ ( (b) PFPh(BipyPr $)_{n}$.

the aromatic protons of Bipy units. As shown in Fig. 1(b), the ${ }^{1} \mathrm{H}$ NMR peaks of $\mathrm{PFPh}(\mathrm{BipyPr})_{n}$ in the low magnetic field region $(\delta$ $=8.0-10.0 \mathrm{ppm})$ are different compared with the ${ }^{1} \mathrm{H}$ NMR peaks of $\mathrm{PFPh}(\text { Bipy })_{n}$ (Fig. 1(a)). Paramagnetic atom aligns with applied external magnetic field and leads to the deshielding effect. Due to the paramagnetic property of Pr, the proton peaks of the ligand may shift and become relatively broad. The proton peaks assigned to the Bipy are broadened and shifted, which may be attributed to the effect of the metal paramagnetic nature. ${ }^{11}$ This indicates that Pr has been incorporated into the polymer by coordination.

The Bipy content in the conjugated polymers was estimated from the ${ }^{1} \mathrm{H}$ NMR spectra, which was based on the ratio between the methylene protons of phthalimide moieties $(\delta=3.55 \mathrm{ppm})$ and the Bipy proton peaks (between $\delta=10.0$ and $8.0 \mathrm{ppm}$ ). The actual values estimated from the ${ }^{1} \mathrm{H}$ NMR are shown in Table 1 . As the feed ratio is increased, so does the actual ratio by an equivalent amount. However, the actual values are lower than the corresponding feed ratio. EDTA coordination reagent has been applied widely in coordination titration to determine the metal element content. Pr content in the copolymers was estimated by EDTA titration after the copolymers were burned in a high temperature first and then dissolved by hydrochloric acid. The results showed that the actual Pr content of polymers was lower than that in the feed ratio. The difference of reaction activity and/or steric hindrance might be responsible for this. To further evidenced the presence of Pr in the polymer, the mass spectra (MALDI-TOF) of PFPh(Bipy) ${ }_{10}$ and PFPh(BipyPr) $)_{10}$ were determined and analyzed. The corresponding spectra are shown in Fig. S3. $\uparrow$ The mass spectrum of PFPh(Bipy) ${ }_{10}$ shows the Bipy moiety fragment ion peaks at $m / z=155-156$. Compared with $\mathrm{PFPh}(\mathrm{Bipy})_{5}$, the mass spectrum of $\operatorname{PFPh}(\text { BipyPr })_{10}$ exhibits a new fragment ion peaks at $\mathrm{m} / \mathrm{z}=293-$ 294, which might indicate the incorporation of Pr complex. The metal inherent nature may be responsible for the high intensity of the peaks at $m / z=293-294$. The number-average molecular weights $\left(M_{\mathrm{n}}\right)$ and weight-average molecular weights $\left(M_{\mathrm{w}}\right)$ of the polymers were measured by GPC in THF solvent as shown in Table 1.

\section{Optical and electrochemical properties}

The UV-vis absorption spectra recorded from thin films are shown in Fig. S4, $\uparrow$ and the results are summarized in Table S1 of the ESI. $\uparrow$ The UV-vis spectra of $\mathrm{PFPh}(\mathrm{Bipy})_{n}$ and $\mathrm{PFPh}(\mathrm{BipyPr})_{n}$ in thin-film were similar and dominated by a single absorption peak at around $363-370 \mathrm{~nm}$, which could be attributed to the $\pi-$ $\pi^{*}$ transition from the conjugated polymer backbone. As Pr was coordinated to the polymer, the absorption peak slightly broadened and the absorption tails extended to a longer

Table 1 Synthetic results of polymerization

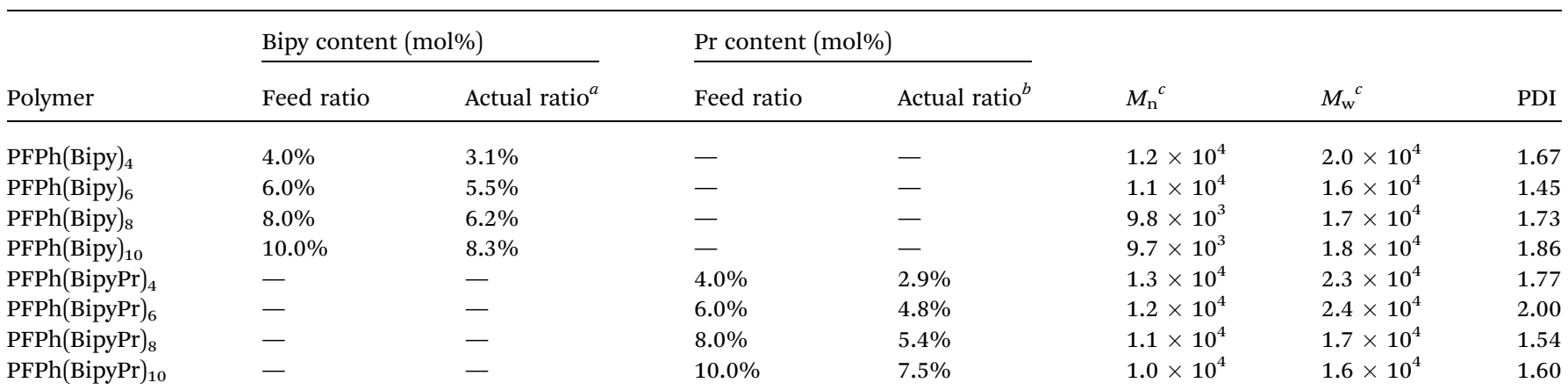

${ }^{a}$ Estimated from the ${ }^{1} \mathrm{H}$ NMR spectroscopy. ${ }^{b}$ Estimated from the ash method and EDTA titration. ${ }^{c}$ Determined by GPC in THF by using a calibration curve of polystyrene standards. 


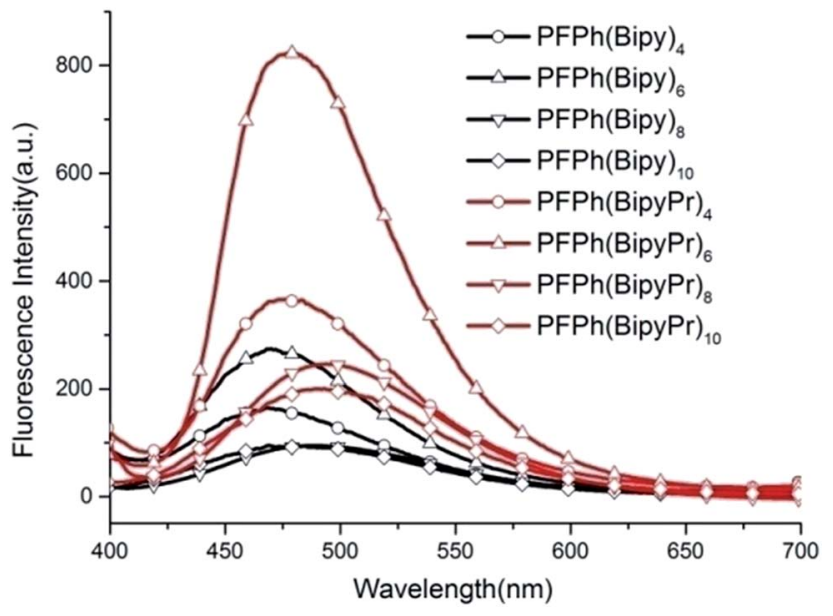

Fig. $2 \mathrm{PL}$ emission spectra of polymers (in film).

wavelength, suggesting the stronger inter-chain interaction due to the Pr atom on the backbone. The optical band gaps of the polymers estimated from the film absorption onset are around 2.48-2.68 eV (shown in Table S1 of the ESI $\dagger$ ). With the increase of Bipy and Pr content in the polymers, the optical band gap of the polymers decreased gradually.

PL spectra recorded from thin films excited at the wavelength of $360 \mathrm{~nm}$ are shown in Fig. 2, and the results are summarized in Table S1 of the ESI. $\dagger$ Compared with that of the Pr-free polymer $\left(\operatorname{PFPh}(\mathrm{Bipy})_{n}\right)$, the emission peaks of the $\mathrm{Pr}$ coordinated polymers $\left(\operatorname{PFPh}(\operatorname{BipyPr})_{n}\right)$ red-shifted and the emission intensity increased obviously. These results indicate that Pr has been incorporated into the polymer by coordination reaction.

Cyclic voltammetry (CV) analysis was used to study the electrochemical behavior of the resulting polymers and to estimate the HOMO and LUMO energy levels.

Fig. 3(a) and (b) show the CV curves of polymers. The results of the electrochemical measurements are listed in Table S1 of the ESI. $\dagger$

The HOMO levels of PFPh(BipyPr $)_{n}$ were $-5.52,-5.57,-5.81$ and $-5.86 \mathrm{eV}$, respectively. The LUMO levels of $\mathrm{PFPh}(\mathrm{BipyPr})_{n}$ were $-2.88,-2.99,-3.29$ and $-3.38 \mathrm{eV}$, respectively. Fig. 3(c) shows the HOMO and LUMO energy levels for PFPh(BipyPr $)_{n}$ along with the work function of the electrodes. Hole injection energy barrier from ITO $(-4.8 \mathrm{eV})$ into HOMO energy level of the Pr-coordinated polymers ( $\left.\mathrm{PFPh}(\mathrm{BipyPr})_{n}\right)$ is $0.72-1.06 \mathrm{eV}$, which is smaller than the electron injection energy barrier between $\mathrm{Al}$ and LUMO level $(0.92-1.42 \mathrm{eV})$ of the polymers. Therefore, $\operatorname{PFPh}(\mathrm{BipyPr})_{n}$ is a p-type semiconductor material, and the memory device behaviors are dominated by hole transportation. The LUMO of Pr-coordinated polymers $\left(\left(\operatorname{PFPh}(\mathrm{BipyPr})_{n}\right)\right)$ are decreased with the increased Pr content. The results indicate that the incorporation of Pr has an influence on the LUMO of the polymers, which was probably responsible for the memory behavior of the polymers.

\section{Electrical switching effects and memory performance}

The memory behaviors of these polymers are demonstrated by the $I-V$ characteristics of ITO/polymer/Al device under an initially positive applied voltage.

The $I-V$ curves of the Pr-free polymers $\left(\mathrm{PFPh}(\mathrm{Bipy})_{n}\right)$ and $\mathrm{Pr}-$ coordinated polymers $\left(\operatorname{PFPh}(\operatorname{BipyPr})_{n}\right)$ memory devices are shown in Fig. 4 and 5. The devices based on PFPh(Bipy) ${ }_{n}$ were kept at a low resistance state during the positive and negative scans, and there was no obvious electrical switching capa-

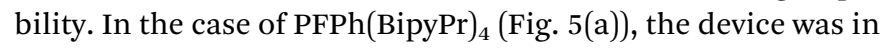
the low conductivity state in the first sweep under the positive voltage, which assigned as OFF state or "0" signal in data storage. However, the current level underwent an abrupt increase at $+1.68 \mathrm{~V}$, which indicated that the device transferred from a low conductivity (OFF) state to a high conductivity (ON) state (sweep 1). This electrical transition represented the writing process of the memory device. The device was kept in the ON state for the subsequent sweep both positive and negative (sweep 2,3). Thus, the device can be used as writeonce-read-many times (WORM) device. But the current radio between the OFF and ON state was only 10, which was insufficient for a low misreading rate of the memory device in actual device applications. For the devices composed of $\operatorname{PFPh}(\mathrm{BipyPr})_{6}, \mathrm{PFPh}(\mathrm{BipyPr})_{8}$ and $\mathrm{PFPh}(\mathrm{BipyPr})_{10}$ (Fig. 5(b)(d)), the $I-V$ curves show similar nonvolatile WORM memory behaviors. In the case of $\operatorname{PFPh}(\operatorname{BipyPr})_{6}$, the current level underwent an abrupt increase at $+1.88 \mathrm{~V}$ under the positive
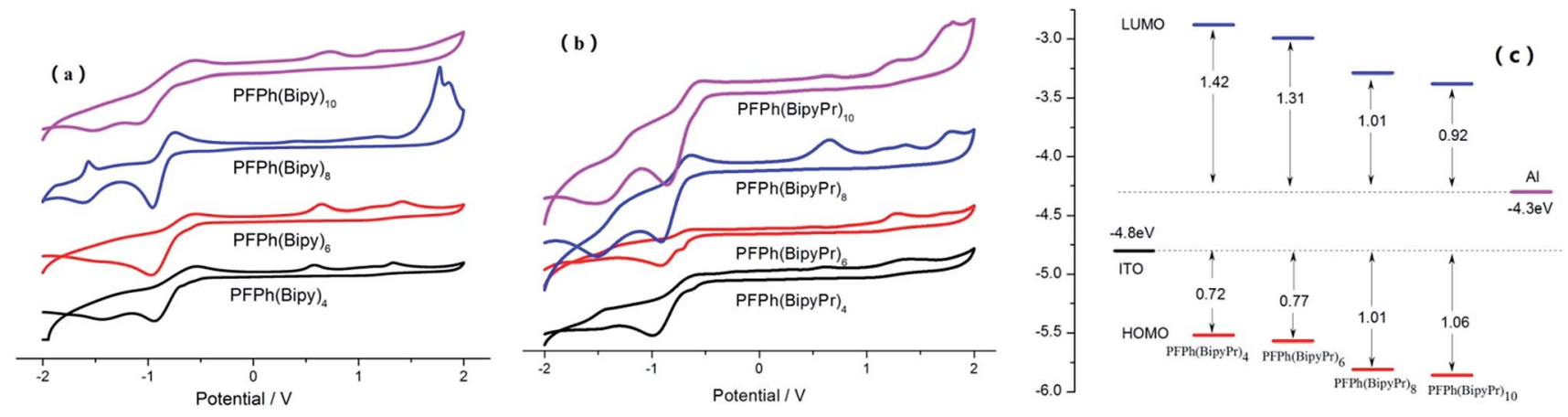

Fig. 3 (a) The CV curves of the polymers PFPh(Bipy) n; (b) the CV curves of the polymers PFPh(BipyPr) ; (c) HOMO and LUMO energy levels for $\mathrm{PFPh}(\mathrm{BipyPr})_{n}$ along with the work function of the electrodes. 

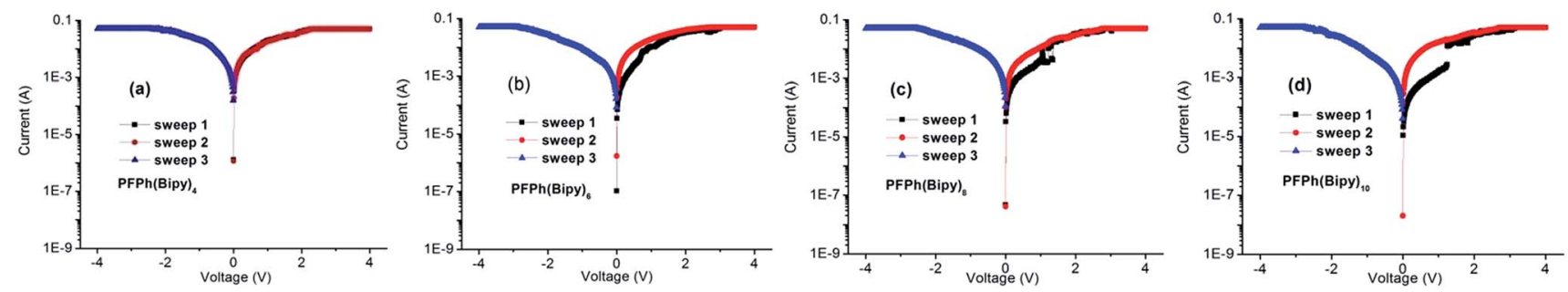

Fig. 4 Current-voltage $(I-V \text { characteristics of the ITO/polymer/Al memory devices based on Pr-free polymers. (PFPh(Bipy) })^{(a)}$ PFPh(Bipy) 4 , (b) $\operatorname{PFPh}(\text { Bipy })_{6}$, (c) PFPh(Bipy) ${ }_{8}$ and (d) PFPh(Bipy) ${ }_{10}$.
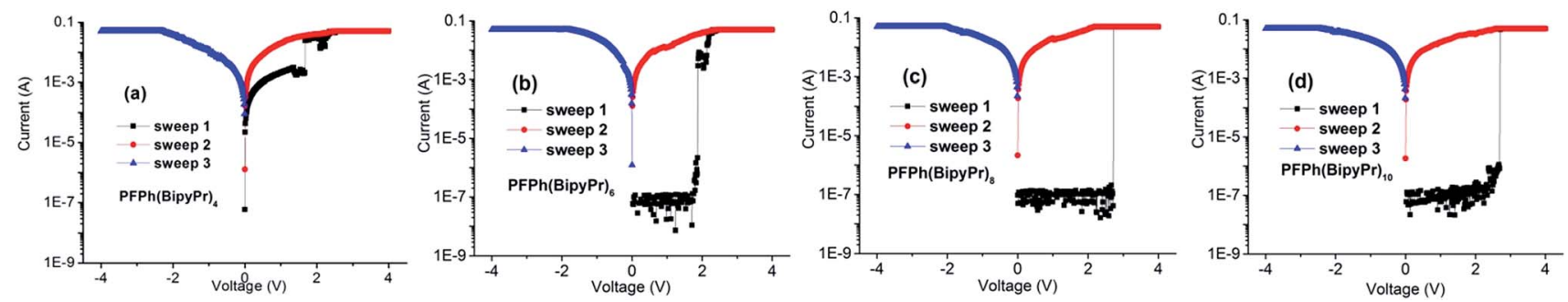

Fig. 5 Current-voltage $(I-V \text { characteristics of the ITO/polymer/Al memory devices based on Pr-coordinated polymers. (PFPh(BipyPr) })^{(a)}$ PFPh(BipyPr) 4 , (b) PFPh(BipyPr) 6 , (c) PFPh(BipyPr) 8 and (d) PFPh(BipyPr) 10 .

voltage, and the current radio between the OFF and ON state was $10^{3}$, which is essential for the memory device to work with the minimal misreading error. As to $\mathrm{PFPh}(\mathrm{BipyPr})_{8}$ and $\operatorname{PFPh}(\operatorname{BipyPr})_{10}$ with the higher Pr content, the threshold voltages were +2.70 and $+2.72 \mathrm{~V}$, while the current radio between the OFF and ON state were about $10^{6}, 10^{5}$, respectively. Notably, the threshold voltages of $\mathrm{PFPh}(\mathrm{BipyPr})_{n}$ were all lower than $3.0 \mathrm{~V}$, which were desirable for low-power memory application.

The parameters of the memory device were measured under ambient conditions. The long-term stability and the stimulus effect of the memory device were evaluated from the retention time and stress tests of both the ON and OFF states of the polymer. Fig. 6(a) shows the stress reliability test measured on the memory device (ITO/PFPh(BipyPr) $\left.)_{6} / \mathrm{Al}\right)$. The ON state current of the memory device remains stable under the continuous voltage stress of $1.0 \mathrm{~V}$ for up to $1800 \mathrm{~s}$. And the OFF state current also exhibits good stability but some slight variations. Fig. 6(b) shows the effect of continuous read pulse of $1.0 \mathrm{~V}$ on the ON and OFF states of the memory device (ITO/PFPh(BipyPr $\left.)_{6} / \mathrm{Al}\right)$. Both the ON and OFF states are stable up to $10^{8}$ read pulse cycles at a read voltage of $1.0 \mathrm{~V}$. For the devices composed of $\mathrm{PFPh}(\mathrm{BipyPr})_{8}$ and $\operatorname{PFPh}(\mathrm{BipyPr})_{10}$, the long-term stability and the stimulus effect of the memory device showed similar behaviors with $\operatorname{PFPh}(\mathrm{BipyPr})_{6}$. Overall, these results suggest that the
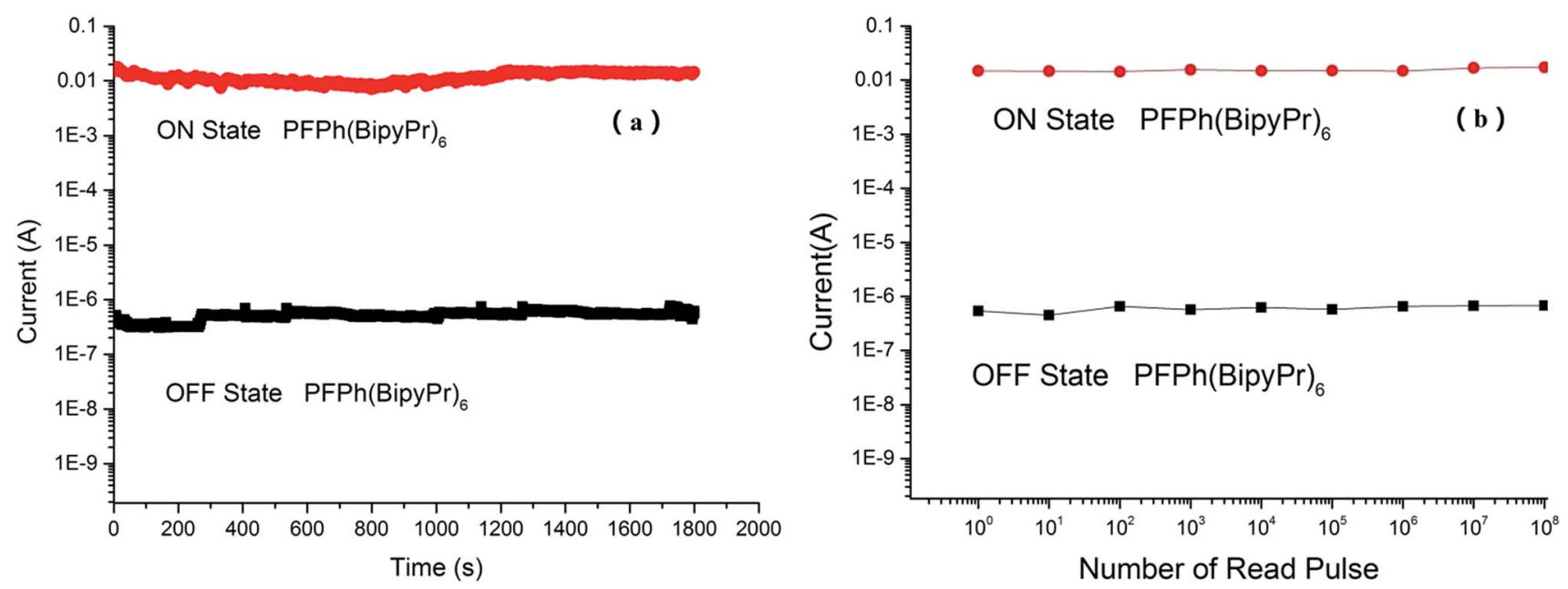

Fig. 6 (a) The retention time for the ON and OFF states of the ITO/PFPh(BipyPr) $6 / \mathrm{Al}$ under a constant bias of $1.0 \mathrm{~V}$; (b) the effect of read pulse applying $1.0 \mathrm{~V}$ on the ON and OFF states of the ITO/PFPh(BipyPr) 6 /Al. 

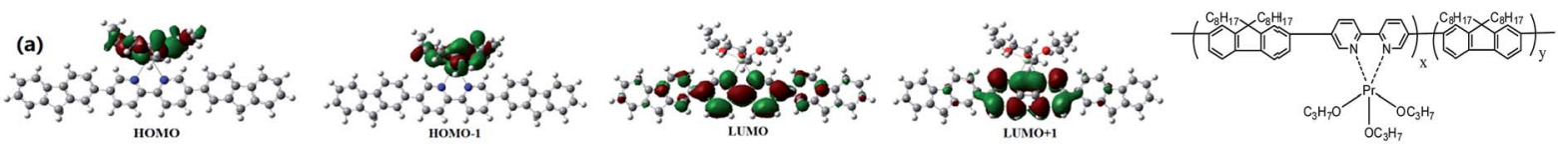

$\operatorname{PF}(\mathrm{BipyPr})_{n}$ омо
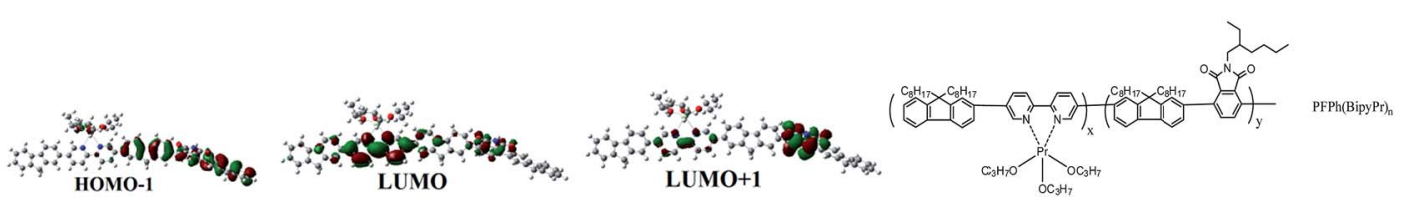

Fig. 7 Calculated HOMO, HOMO - 1 and LUMO, LUMO + 1 of the model oligomer of (a) PF(BipyPr) ${ }_{n}$ ( (b) PFPh(BipyPr) .

memory devices possessed good reproducibility and stability.

For comparison, the similar device based on polymer without $\mathrm{Ph}$ moiety $\mathrm{PF}(\text { BipyPr })_{6}$, was also fabricated. $\mathrm{PF}(\mathrm{BipyPr})_{6}$ does not exhibit obvious memory behaviors. The corresponding structure was shown in ESI. $\dagger$ Liu et al. ${ }^{\mathbf{1 4}}$ reported that conjugated polymers consisting of fluorene and an Ir(III) complex exhibited the rewritable (FLASH) memory effect. Compared with their work, the memory device based on the polymer $\mathrm{PF}(\text { BipyPr })_{6}$ in the $I-V$ characteristics shows different behavior and has not memory effect, which might be related to the neutral Pr atom and triisopropoxide groups. However, by the introduction of $\mathrm{Ph}$ moiety with proper electron-accepting ability, the device based on the polymer $\operatorname{PFPh}(\mathrm{BipyPr})_{n}$ exhibits the WORM memory behavior.

\section{Possible mechanism of memory behavior}

The results show that the Pr-free polymers PFPh(Bipy) ${ }_{n}$ do not exhibit electronic bistability and memory effects and the neutral Pr complexes might play the key role in the memory behavior of polymers PFPh(BipyPr $)_{n}$. It is well-known that the lanthanide complexes have a high and variable coordination number. Thus, Pr complex is an electron-deficient complex and can act as an electron acceptor, which can serve as a deep trap center in the electric field. ${ }^{\mathbf{1 2 , 1 7}}$ In addition, the incorporation of Pr complexes to the polymers might have some effect on the molecular stacking of the polymers, which leads to promote the polymers to form partial random structure at the ground state, resulting in low-conductivity state at a low field. The devices based on $\operatorname{PFPh}(\text { BipyPr })_{n}$ were in their lowconductivity state in the low voltage range during the first sweep since there were not much free charge carriers in the polymers before the traps were filled. When the applied voltage was high enough, the traps in the polymer became

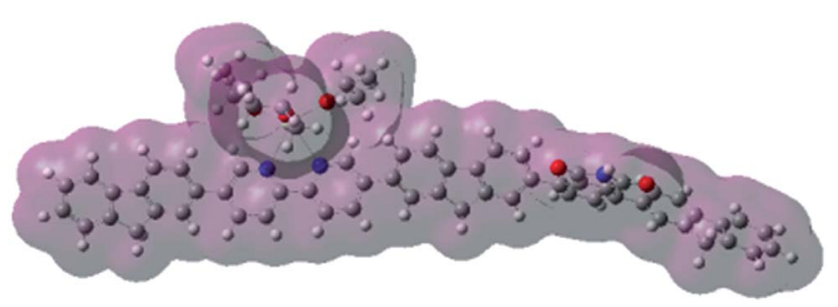

Fig. 8 ESP surface of PFPh(BipyPr) . filled and the device showed a high current. Furthermore, the formation of a charge-transfer (CT) state in the polymers might be responsible for the ON state. After the power was turn off, the ON state can still remain due to the stability of the CT complex.

The memory behavior may be attributed to the CT state between the electron donor unit and the electron accepter unit of the polymer. To better understand the mechanism of the memory device, the molecular orbital and electronic properties of the model oligomers of $\mathrm{PF}(\mathrm{BipyPr})_{n}$ without $\mathrm{Ph}$ moiety and $\mathrm{PFPh}(\mathrm{BipyPr})_{n}$ were explored by density functional theory (DFT) calculations. Fig. 7 shows the calculated HOMO, HOMO -1 , LUMO and LUMO +1 of the model oligomers of $\operatorname{PF}(\text { BipyPr })_{n}, \operatorname{PFPh}(\text { BipyPr })_{n}$. As depicted in Fig. 7(a), the calculated HOMO, HOMO - 1 isosurfaces for the model oligomer of $\mathrm{PF}(\mathrm{BipyPr})_{n}$ are located on the triisopropyloxide moiety and the LUMO, LUMO + 1 isosurfaces are located on the Bipy moiety. Nevertheless, the Bipy and triisopropyloxide proportions of the polymer $\mathrm{PF}(\mathrm{BipyPr})_{n}$ were very small, so a steady CT state might not form in the main chain of the polymer. As shown in Fig. 7(b), the calculated HOMO, HOMO -1 isosurfaces for the model oligomer of $\mathrm{PFPh}(\mathrm{BipyPr})_{n}$ are located on the triisopropyloxide moiety and fluorene moiety, the LUMO, LUMO + 1 isosurfaces are located on the Bipy moiety and $\mathrm{Ph}$ moiety. By the incorporation of Ph moiety, the fluorene moiety on the main chain might serve as the electron donor unit and the $\mathrm{Ph}$ moiety as the electron acceptor unit. The distinct asymmetric distribution of HOMO and LUMO indicates that charge-transfer processes can occur when the polymer is activated by electrical excitation leading to forming a CT state. Moreover, a channel is formed from the molecular surface throughout the polymer backbone with continuous molecular electrostatic potential (ESP in Fig. 8), which is beneficial for the migration of charge carriers along the polymer backbone. As shown in Fig. 8, negative ESP regions (dark region) located at the Pr center can be observed, indicating that the Pr complexes can act as "traps" to block the mobility of charge carriers. ${ }^{17}$ The depth of these trap, which arises from the Pr complexes, might affect the memory performance of the devices. The deep traps may bring about non-volatile memory behavior because filled traps can't easily be de-trapped. The appropriate trap depth and the formation of stable CT state should be responsible for good non-volatile memory behavior, such as high ON/OFF current ratio and excellent stability. 


\section{Conclusions}

In summary, a series of novel Pr-coordinated conjugated polymers containing neutral Pr complexes were successfully synthesized by the Suzuki coupling reaction and coordination reaction. The content of Pr can be easily adjusted by changing the Bipy unit proportion of the polymers. The resulting polymers were further studied in the polymer memory devices. By the introduction of $\mathrm{Ph}$ moiety with proper electron-accepting ability, the devices based on the Pr-coordinated polymers with higher Pr content exhibit the function of non-volatile WORM memory with a high ON/OFF current ratio and excellent stability, which cast lights on the coordinated-polymers containing Pr complexes materials used in polymer memory devices. As far as we know, this is the first report about the memory behaviors of polymers containing neutral $\mathrm{Pr}$ complexes. This work should be helpful for the further design and development of memory materials based on polymers containing lanthanide metal complexes.

\section{Acknowledgements}

This work was financially supported by the National Natural Science Foundation of China (Grant No. 21574021, 51573026, 21401023), the Natural Science Foundation of Fujian Province (Grant No. 2015J01189), the Program for Innovative Research Team in Science and Technology in Fujian Province (IRTSTFJ) and the Program of the Education Department of Fujian Province (Grant No. JA14078).

\section{References}

1 Y. Yang, J. Y. Ouyang, L. P. Ma, R. J. H. Tseng and C. W. Chu, Adv. Funct. Mater., 2006, 16, 1001.

2 S. H. Jo and W. Lu, Nano Lett., 2008, 8, 392.

3 Q. Ling, D. Liaw, C. Zhu, D. S. Chan, E. Kang and K. Neoh, Prog. Polym. Sci., 2008, 33, 917.

4 P. Heremans, G. H. Gelinck, R. Müller, K. J. Baeg, D. Y. Kim and Y. Y. Noh, Chem. Mater., 2011, 23, 341.

5 W. Lin, S. Liu, T. Gong, Q. Zhao and W. Huang, Adv. Mater., 2014, 26, 570.

6 G. Liu, Q. Ling, E. Y. H. Teo, C. Zhu, D. S. Chan, K. Neoh and E. Kang, ACS Nano, 2009, 3, 1929.

7 S. Lian, C. Liu and W. Chen, ACS Appl. Mater. Interfaces, 2011, 3, 4504.

8 C. Wang, P. Gu, B. Hu and Q. Zhang, J. Mater. Chem. C, 2015, 10055.

9 T. Kurosawa, T. Higashihara and M. Ueda, Polym. Chem., 2013, 16.

10 Y. Ko, S. G. Hahm, K. Murata, Y. Y. Kim, B. J. Ree, S. Song, T. Michinobu and M. Ree, Macromolecules, 2014, 47, 8154.

11 Y. Song, Y. P. Tan, E. Y. H. Teo, C. Zhu, D. S. H. Chan, Q. D. Ling, K. G. Neoh and E. T. Kang, J. Appl. Phys., 2006, 8, 84508.
12 Q. D. Ling, W. Wang, Y. Song, C. X. Zhu, D. S. H. Chan, E. T. Kang and K. G. Neoh, J. Phys. Chem. B, 2006, 110, 23995.

13 S. Choi, S. H. Hong, S. H. Cho, S. Park, S. M. Park, O. Kim and M. Ree, Adv. Mater., 2008, 20, 1766.

14 S. J. Liu, Z. H. Lin, Q. Zhao, Y. Ma, H. F. Shi, M. D. Yi, Q. D. Ling, Q. L. Fan, C. X. Zhu, E. T. Kang and W. Huang, Adv. Funct. Mater., 2011, 21, 979.

15 S. J. Liu, W. P. Lin, M. D. Yi, W. J. Xu, C. Tang, Q. Zhao, S. H. Ye, X. M. Liu and W. Huang, J. Mater. Chem., 2012, 22, 22964.

16 S. J. Liu, P. Wang, Q. Zhao, H. Y. Yang, J. Wong, H. B. Sun, X. C. Dong, W. P. Lin and W. Huang, Adv. Mater., 2012, 24, 2901.

17 P. Wang, S. J. Liu, Z. H. Lin, X. C. Dong, Q. Zhao, W. P. Lin, M. D. Yi, S. H. Ye, C. X. Zhu and W. Huang, J. Mater. Chem., 2012, 22, 9576.

18 W. P. Lin, H. B. Sun, S. J. Liu, H. R. Yang, S. H. Ye, W. J. Xu, Q. Zhao, X. M. Liu and W. Huang, Macromol. Chem. Phys., 2012, 213, 2472.

19 A. Bandyopadhyay, S. Sahu and M. Higuchi, J. Am. Chem. Soc., 2011, 133, 1168.

20 T. L. Choi, K. H. Lee, W. J. Joo, S. Lee, T. W. Lee and M. Y. Chae, J. Am. Chem. Soc., 2007, 129, 9842.

21 J. Xiang, T. Wang, Q. Zhao, W. Huang, C. Ho and W. Wong, J. Mater. Chem. C, 2016, 4, 921.

22 B. Cui, Z. Mao, Y. Chen, Y. Zhong, G. Yu, C. Zhan and J. Yao, Chem. Sci., 2015, 6, 1308-1315.

23 N. D. Paul, U. Rana, S. Goswami, T. K. Mondal and S. Goswami, J. Am. Chem. Soc., 2012, 134, 6520.

24 E. Y. Hong, C. Poon and V. W. Yam, J. Am. Chem. Soc., 2016, 138, 6368-6371.

25 V. K. Au, D. Wu and V. W. Yam, J. Am. Chem. Soc., 2015, 137, 4654-4657.

26 C. Jin, J. Lee, E. Lee, E. Hwang and H. Lee, Chem. Commun., 2012, 48, 4235.

27 Q. D. Ling, E. T. Kang, K. G. Neoh and W. Huang, Macromolecules, 2003, 36, 6995.

28 Q. D. Ling, M. J. Yang, Z. F. Wu and W. Zhang, Polymer, 2001, 42, 4605.

29 L. Wang, W. Wang, W. Zhang, E. Kang and W. Huang, Chem. Mater., 2000, 12, 2212.

30 L. Wang and W. Zhang, Chem. J. Chin. Univ., 1996, 17, 808. 31 T. Huang, C. Tsai, S. Hsiao and G. Liou, $R S C A d v ., 2016,6$, 28815.

32 M. J. Frisch, et al., Gaussian 09, revision A.2, Gaussian, Inc., Wallingford CT, 2009.

33 L. Maron and O. Eisenstein, J. Phys. Chem. A, 2000, 104, 7140. 34 M. Dolg, H. Stoll, A. Savin and H. Preuss, Theor. Chim. Acta, 1989, 75, 173.

35 X. Guo, F. S. Kim, S. A. Jenekhe and M. D. Watson, J. Am. Chem. Soc., 2009, 131, 7206. 